\title{
PET scan findings can be false positive
}

\author{
E. Safaie ${ }^{1} \cdot$ R. Matthews ${ }^{1} \cdot$ R. Bergamaschi ${ }^{2}$
}

Received: 19 March 2015/Accepted: 14 April 2015/Published online: 5 May 2015

(C) Springer-Verlag Italia Srl 2015

Positron emission tomography-computed tomography (PET-CT) scans with [18F]-fluorodeoxyglucose (FDG) and PET-magnetic resonance imaging (MRI) have become standard practice in staging and restaging of colorectal cancer patients by providing important information about the primary cancer as well as metastases. The PET portion of this imaging modality relies on the accumulation of radioactive glucose analog, FDG. In cancer cells, there is an overproduction of glucose transporters and, as a result, increased FDG uptake. However, not all PET-positive lesions are cancer, and in many instances, PET findings can be false positive.

A few points need to be considered before understanding FDG. First, not all cancer cells use the same amount of glucose: some use more and some use less. Cancer cells with a faster metabolic rate such as colorectal adenocarcinoma are very FDG avid, whereas others such as mucinous cancers consume less glucose and therefore are less FDG avid. Inflammatory cells also have increased metabolic rates and, as a result, are FDG avid.

Many of us have had patients or know of patients who were treated by the medical oncologist for stage IV cancer only to find out what was assumed to be a metastatic lesion was benign on pathology. Other patients have undergone multiple biopsies of supposed metastatic mesenteric lymph nodes that subsequently turned out to be fat necrosis or a

R. Bergamaschi

rcmbergamaschi@gmail.com

1 Department of Radiology, State University of New York, Stony Brook, NY, USA

2 Division of Colon and Rectal Surgery, Health Science Center T18, Suite 046B, State University of New York, Stony Brook, NY 11794-8191, USA granulomatous reaction. FDG-positive lesions often mean cancer, but not always. A variety of lesions have increased FDG radiotracer including infection, inflammation, autoimmune processes, sarcoidosis, and benign tumors. If such conditions are not identified accurately and in a timely manner, misdiagnosis can lead to inadequate therapies.

Within the lower gastrointestinal tract, physiological FDG uptake can be highly variable and may range from mild to intense with a focal, diffuse, or segmental distribution. To add to the confusion, swallowed secretions, lymphoid tissue uptake, microbial uptake, infection, and benign tumors show increased FDG uptake. For example, incidental focal FDG uptake in the colon or rectum has only a $47 \%$ probability of showing an underlying adenoma or malignant lesion on colonoscopy [1].

It is well documented that surgical staples or suture lines following bowel resection can cause inflammatory or granulomatous changes with scar tissue formation with increased FDG uptake [2]. Often the only way to differentiate between benign and malignant pathology in this setting is by colonoscopy with biopsy. Chemotherapy and surgery itself can produce reactive lymphadenopathy. Radiation therapy can produce an intense inflammatory response that often develops months after treatment with reactive lymphadenopathy, peritoneal changes, and mass development. These changes can appear similar to cancer with increased FDG uptake that can last years after the cessation of therapy.

Imaging with a complex technology as with PET-CT or PET-MRI can create a multitude of artifacts confounding diagnosis. PET-CT and PET-MRI use an attenuation-based correction that causes dense materials such as metal, IV contrast, or barium to artificially increased signals on the PET image. Variable gas in the bowel can cause image attenuation artifacts leading to false areas of increased 
uptake. The bladder with a high concentration of FDG can produce a "shine through" artifact causing PET signal distortion adjacent to the bladder.

There are many false-positive causes of FDG uptake. The pattern of FDG uptake is easily identifiable as benign or malignant, especially if this is confirmed by CT or MRI findings. However, there are still a considerable number of challenging PET findings that may lead to false interpretation with subsequent inappropriate treatment decisions. When seeing a new focus of FDG uptake, before informing the patient that the lesion is cancer, the surgeon and oncologist must determine whether this is truly a cancer or a possible false-positive finding.
Conflict of interest None.

\section{References}

1. van Hoeij FB, Keijsers RG, Loffeld BC, Dun G, Stadhouders PH, Weusten BL (2015) Incidental colonic focal FDG uptake on PET/ CT: can the maximum standardized uptake value (SUVmax) guide us in the timing of colonoscopy? Eur J Nucl Med Mol Imaging 42:66-71

2. Shyn PB, Madan R, Wu C, Erturk SM, Silverman SG (2010) PET/ CT pattern analysis for surgical staple line recurrence in patients with colorectal cancer. AJR Am J Roentgenol 194:414-421 